\title{
Oxygen therapy alleviates hepatic steatosis by inhibiting hypoxia-inducible factor-2 $\alpha$
}

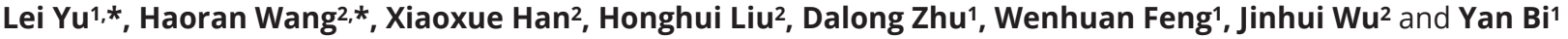 \\ 1Department of Endocrinology, Drum Tower Hospital Affiliated to Nanjing University Medical School, Nanjing, China \\ 2State Key Laboratory of Pharmaceutical Biotechnology, Medical School, Nanjing University, Nanjing, China
}

Correspondence should be addressed to W Feng or J Wu or Y Bi: fengwh501@163.com or wuj@nju.edu.cn or biyan@nju.edu.cn

*(L Yu and $\mathrm{H}$ Wang contributed equally to this work)

\begin{abstract}
Non-alcoholic fatty liver disease (NAFLD) is difficult to manage due to the lack of effective treatments. Increased oxygen consumption caused by overnutrition, along with reduced oxygen delivery to liver cells induces hepatic steatosis. Here, we investigated the efficacy of oxygen therapy (OT) to alleviate hepatic steatosis. The effect of OT on hepatic steatosis was evaluated in high-fat-diet (HFD)-fed mice and palmitic acid (PA)treated primary hepatocytes. Liver biopsy tissue samples were used to determine the relationship between the expression of hypoxia-inducible factor-2 $\alpha$ (HIF- $2 \alpha)$ and the progression of NAFLD. The role of HIF-2 $\alpha$ in the OT group was determined based on the overexpression of HIF-2 $\alpha$ in vitro. OT safely alleviated hepatic hypoxia and improved hepatic steatosis by inhibiting hepatic de novo lipogenesis in HFD-fed mice and PA-treated primary hepatocytes, and this was accompanied by reduced expression of HIF-2 $\alpha$ and hepatic de novo lipogenesis. The analysis of liver tissues from individuals with or without NAFLD revealed a positive correlation between hepatic HIF-2 $\alpha$ expression and NAFLD progression. Overexpression of HIF- $2 \alpha$ in vitro inhibited the beneficial effect of OT against hepatic lipogenesis and steatosis. OT might be a viable treatment option for NAFLD and functions by alleviating hypoxia and inhibiting the liver HIF- $2 \alpha$ signaling pathway.
\end{abstract}
Key Words
- HIF- $2 \alpha$
- NAFLD
- hepatic steatosis
- oxygen therapy

\section{Introduction}

Non-alcoholic fatty liver disease (NAFLD), a major epidemic chronic liver disease worldwide, is often accompanied by systemic metabolic disorders, such as obesity, type 2 diabetes and cardiovascular diseases (Byrne \& Targher 2015, Pais et al. 2016). The global average prevalence of NAFLD in adults is $25 \%$, and $15 \%$ of these patients might progress from simple steatosis to non-alcoholic steatohepatitis (NASH), increasing the risk of cirrhosis and hepatocellular carcinoma (Febbraio et al. 2019). NASH is predicted to become the primary indication for liver transplantation in Western countries
(Cohen et al. 2011). Thus, safe and effective treatments for NAFLD are urgently required.

The advocate treatment for NAFLD is weight loss through lifestyle modifications consisting of diet and exercise (Romero-Gomez et al. 2017). Weight loss of 3-5\% reduces steatosis, and further weight loss ( $\geq 5-7 \%)$ has been shown to induce the remission of NASH, whereas sustained weight loss of $10 \%$ helps to improve hepatic fibrosis (Hannah \& Harrison 2016). However, poor compliance often makes it difficult to maintain this long term. Although therapies targeting various pathogenic features 
of NAFLD (insulin resistance, hepatic fat accumulation, free radical oxidative stress, inflammation, microbiota and fibrosis) have been widely reported, currently, there is no approved pharmacological treatment to effectively alleviate NAFLD (Rotman \& Sanyal 2017).

High oxygen consumption (evidenced by increased mitochondrial respiratory rates due to nutrient overload) and reduced oxygen delivery to liver cells (induced by steatosis) are crucial pathophysiological mechanisms underlying the occurrence and development of NAFLD (Mantena et al. 2009, Koliaki et al. 2015, Anavi et al. 2017). A physiologic oxygen gradient exists in healthy livers, resulting in the metabolic zonation of hepatic lobules and causing hepatocytes in different regions to participate in different types of lipid metabolism to avoid excessive hepatic lipid droplet deposition. However, destruction of the oxygen gradient by hypoxia accelerates hepatic steatosis (Birchmeier 2016, Kietzmann 2017, 2019). Hypoxia-inducible transcription factors (HIFs) are heterodimers that mediate the hypoxia response in the liver, and they are composed of a regulatory HIF- $\alpha$ subunit (HIF-1 $\alpha$, HIF- $2 \alpha$ and HIF-3 $\alpha$ ) and a structural HIF- $1 \beta$ subunit (HIF-1, HIF-2 and HIF-3) (Koh \& Powis 2012).

Experimental studies have indicated that HIF- $2 \alpha$ might be a therapeutic target for NAFLD, as the activation of HIF- $2 \alpha$ (and not HIF-1 $\alpha$ ) leads to NAFLD (Qu et al. 2011). However, recent studies have suggested that the pharmacological inhibition of HIF- $2 \alpha$ is not a suitable therapy for NAFLD because of the increased risk of elevated postprandial blood glucose, which would aggravate type 2 diabetes (Wei et al. 2013, Ramakrishnan et al. 2016, Ramakrishnan \& Shah 2017). Although hypoxia and increased HIF- $2 \alpha$ expression induce hepatic steatosis, it is unclear whether oxygen therapy (OT) can help to alleviate NAFLD. We conducted this study to determine the efficacy of OT in improving NAFLD in vivo and in vitro and to uncover the role of HIF- $2 \alpha$ in OT.

\section{Materials and methods}

\section{Oxygen therapy}

An oxygen chamber containing two small holes and a door was sterilised with $75 \%$ ethanol. The OT group of mice or cells was then moved into the oxygen chamber. The oxygen chamber was ventilated through one of the holes with a pipe connected to $100 \%$ oxygen for $1 \mathrm{~min}$, and then the door of the oxygen chamber was closed and the other hole was connected to the outside. The oxygen concentration in the chamber was maintained between $88 \%$ and $100 \%$ using an oxygen meter based on the reading (Oxygen Meter QD-1100; ESC, Boca Raton, FL, USA).The animal group was subjected to OT at $88 \%$ oxygen concentration for $1 \mathrm{~h}$ each day at 08:00 $\mathrm{h}$ and 20:00 $\mathrm{h}$, and the cell group was subjected to OT at 100\% oxygen concentration for $1 \mathrm{~h}$ (once).

\section{Animal experimentation}

Four-week-old male mice with the C57BL/6J genetic background were obtained from the Model Animal Research Center of Nanjing University, Nanjing, China. The mice were housed in colony cages with $12 \mathrm{~h}$ light:12 $\mathrm{h}$ darkness cycles, and they had free access to food and water. The study diets were a high-fat diet (HFD: 60\% fat, $20 \%$ protein and 20\% carbohydrates; Research Diets, Inc., New Brunswick, NJ, USA) and normal chow diet (CON: $65 \%$ carbohydrate, $24 \%$ protein and $11 \%$ fat; MediScience Diets Co. Ltd, Yangzhou, China).

After 1 week of adaptation to a normal chow diet, the mice were randomly divided into two groups and fed either the CON $(n=7)$ or HFD $(n=14)$. After 4 weeks, the mice in the HFD group were randomly divided into the HFD $(n=7)$ and OT groups $(n=7)$, and the mice in the OT group were provided both HFD and OT (88\% oxygen concentration, 1 atm, bid, $1 \mathrm{~h}$ each time) for 8 weeks. In the last 2 weeks, the mice were intraperitoneally injected with glucose $(2 \mathrm{~g} / \mathrm{kg}$ body weight) and insulin (1 U/kg body weight) for glucose tolerance testing (GTT) and insulin tolerance testing (ITT), respectively, after fasting for $8 \mathrm{~h}$. Blood samples were collected from the tail veins of mice before and at 30, 60 and $120 \mathrm{~min}$ after the injection. All animals were anesthetised and killed in an isoflurane-filled room 2 days after ITT. The blood oxygen concentration at the outlet of the inferior vena cava near the liver was measured in three anesthetised mice per group, and the mice in the OT group were administered OT before blood oxygen detection. The blood oxygen concentration was measured using an oxygen probe (OX-NP; a $1.6 \times 40 \mathrm{~mm}$-needle sensor for piercing, Unisense A/S Co., Ltd., Aarhus, Denmark). All animal experiments were performed in accordance with the guidelines and approved by the Institutional Animal Care and Use Committee of Nanjing University.

\section{Primary hepatocyte isolation and culture}

Primary hepatocytes were isolated from 4-week-old male mice using a modified two-step collagenase perfusion method. The inferior vena cava of the anesthetised mice was perfused with $40 \mathrm{~mL}$ of Hank's Balanced Salt Solution 
(Beyotime, Shanghai, China) for $6 \mathrm{~min}$, and then with 30 $\mathrm{mL}$ of Hanks' Balanced Salt Solution with $\mathrm{Ca}^{2+}$ and $\mathrm{Mg}^{2+}$ (Beyotime) containing collagenase IV $(0.2 \mathrm{mg} / \mathrm{mL}$, Cat. no. 17104019; Gibco) for $5 \mathrm{~min}$. The liver was removed, rinsed with PBS and then placed in $10 \mathrm{~mL}$ of Hanks' Balanced Salt Solution with $\mathrm{Ca}^{2+}$ and $\mathrm{Mg}^{2+}$, shredded, and filtered through a sieve and funnel. The filtrate was added to a 50-mL pre-cooled centrifuge tube and supplemented with $20 \mathrm{~mL}$ of Dulbecco's modified Eagle's medium (DMEM) at $4^{\circ} \mathrm{C}$ and centrifuged at $50 \boldsymbol{g}$ for $2 \mathrm{~min}$, and the pellet was resuspended in DMEM, and this step was repeated three times. Subsequently, $15 \mathrm{~mL}$ of pre-chilled medium was added to resuspend the pellet.

The viability of hepatocytes was validated based on trypan blue uptake, and cells with more than $85 \%$ viability were used in the subsequent experiments. Hepatocytes were seeded into a six-well plate and cultured in DMEM with $10 \%$ fetal bovine serum, $100 \mathrm{U} / \mathrm{mL}$ penicillin and $100 \mu \mathrm{g} / \mathrm{mL}$ streptomycin. After $4-6 \mathrm{~h}$ of seeding at $37^{\circ} \mathrm{C}$ under 5\% $\mathrm{CO}_{2}$ in 95\% air, humidified atmosphere, the DMEM was changed according to the cell growth conditions. After the adherence of hepatocytes to the wall, they were divided into the following three groups: CON, PA (palmitic acid; Sigma) and OT. PA (0.25 mM) was administered for $24 \mathrm{~h}$, and the OT group was administered oxygen $(100 \%$ oxygen, $1 \mathrm{~atm})$ in the last hour of PA intervention.

For HIF- $2 \alpha$ overexpression studies, the hepatocytes were divided into the following four groups: PA, OT, lentivirus (LV)-green fluorescent protein $(\mathrm{GFP})+\mathrm{OT}$ and LV-HIF-2 $\alpha+$ OT. LV-GFP or LV-HIF-2 $\alpha$ (Genechem, Shanghai, China) was transfected into LV-GFP+OT or LV-HIF- $2 \alpha+$ OT group cells, respectively, at $12 \mathrm{~h}$ after seeding. At $48 \mathrm{~h}$ post transfection, all the four groups were treated with PA for $24 \mathrm{~h}$, and except for the PA group, the other three groups were subjected to OT in the last hour. The cells were collected for Western blotting and real-time PCR analysis.

\section{H\&E and Oil Red O staining}

After excising the liver, some tissues were fixed with $4 \%$ paraformaldehyde and frozen in liquid nitrogen. Paraformaldehyde-fixed tissues were embedded in paraffin. The prepared paraffin sections were dewaxed in water, washed with distilled water, soaked in hematoxylin stain for $10 \mathrm{~min}$ and then rinsed with distilled water to remove excess stain solution. The specimens were rinsed with flowing distilled water for approximately $20 \mathrm{~min}$. After soaking in eosin stain for $10 \mathrm{~min}$, excess eosin was removed by rinsing with distilled water. The samples were treated with an increasing alcohol gradient $(70 \%$, $80 \%$ and $90 \%$ ) for dehydration, followed by sealing with gelatine after routine dehydration.

The frozen tissue was sectioned, fixed with formaldehyde fixing solution for $20 \mathrm{~min}$, washed with distilled water 2-3 times, stained with Oil Red $\mathrm{O}$ dye solution for $20 \mathrm{~min}$ and immersed in $70 \%$ ethanol. After rinsing with distilled water, the sections were counterstained with hematoxylin for approximately 8-10 s. Finally, the sections were sealed with gelatine. The images were captured using the Panoramic 250 slide scanner (3DHistech, Ltd., Budapest, Hungary).

\section{Human subjects}

According to the guidance of the Nation Health and Medical Research Council of China, the study with humans was approved by the Ethics Committee of Nanjing Drum Tower Hospital, Nanjing University Medical School. All subjects provided informed consent. The study protocol was performed in accordance with the ethical guidelines of the 1975 Helsinki Declaration. This study has been registered with ClinicalTrials.gov (No. NCT03296605). Frozen liver tissue samples $(1.5 \times 2.0 \times 2.0$ $\left.\mathrm{cm}^{3}\right)$ were obtained from patients with NAFLD $(n=14)$ who underwent weight-loss metabolic surgery (Roux-en-Y gastric bypass) and from those with hepatic hemangioma without NAFLD $(n=7)$. Fourteen patients with NAFLD were divided into the non-alcoholic simple fatty liver (NAFL) group $(n=7)$ and the NASH group $(n=7)$ based on their NAFLD activity score (NAS), assessed by a pathologist uninformed of the present study. The liver samples were immediately frozen and stored at $-80^{\circ} \mathrm{C}$ until immunohistochemistry and real-time PCR analyses, and patients with hepatitis, cirrhosis, liver cancer or chronic alcohol use were excluded. The clinical and biochemical characteristics of the patients are presented in Table 1.

\section{Biochemical analyses and oxidative stress tests}

Cell counting kit-8 (CCK8; Dojindo Laboratories, Kumamoto, Japan) was used for the growth inhibition assay. Total cholesterol, triglycerides (TGs) and highand low-density lipoprotein cholesterol in mice were measured using ELISA kits according to the manufacturer's instructions (Colorfulgene Biological Technology, Ltd., Wuhan, China). Alanine aminotransferase, aspartate aminotransferase, gamma glutamyltransferase, total cholesterol, TG, high- and low-density lipoprotein 
Table 1 Clinical and biochemical characterisation of patients investigated.

\begin{tabular}{l}
\hline Parameters \\
\hline NAS \\
Female/male \\
Age (year) \\
BMI $\left(\mathrm{kg} / \mathrm{m}^{2}\right)$ \\
SBP $(\mathrm{mmHg})$ \\
DBP $(\mathrm{mmHg})$ \\
FBG $(\mathrm{mmol} / \mathrm{L})$ \\
ALT $(\mathrm{U} / \mathrm{L})$ \\
AST $(\mathrm{U} / \mathrm{L})$ \\
LDH $(\mathrm{U} / \mathrm{L})$ \\
$\gamma$-GT $(\mathrm{U} / \mathrm{L})$ \\
TG (mmol/L) \\
TC (mmol/L) \\
HDL (mmol/L) \\
LDL (mmol/L) \\
Apolipoprotein A (g/L) \\
Apolipoprotein B (g/L) \\
\hline
\end{tabular}

\begin{tabular}{c}
\hline Non-NAFLD $(n=7)$ \\
\hline 0 \\
$4 / 3$ \\
$53.9 \pm 8.6$ \\
$23.5 \pm 2.5$ \\
$129.9 \pm 21.0$ \\
$82.1 \pm 10.2$ \\
$4.8 \pm 0.7$ \\
$19.3 \pm 4.9$ \\
$19.2 \pm 4.9$ \\
$172.4 \pm 25.0$ \\
$26.8 \pm 10.3$ \\
$1.4 \pm 0.9$ \\
$4.6 \pm 0.8$ \\
$1.2 \pm 0.3$ \\
$2.7 \pm 0.6$ \\
$1.2 \pm 0.2$ \\
$0.9 \pm 0.2$
\end{tabular}

\begin{tabular}{c}
\hline NAFL $(n=7)$ \\
\hline $1.1 \pm 0.4$ \\
$6 / 1$ \\
$33.0 \pm 9.9$ \\
$32.0 \pm 2.3$ \\
$129.4 \pm 18.4$ \\
$84.6 \pm 14.4$ \\
$5.4 \pm 1.2$ \\
$18.9 \pm 8.4$ \\
$16.7 \pm 4.5$ \\
$174.6 \pm 21.7$ \\
$35.4 \pm 18.1$ \\
$3.1 \pm 2.2$ \\
$2.9 \pm 1.8$ \\
$2.0 \pm 1.2$ \\
$1.9 \pm 0.7$ \\
$1.2 \pm 0.3$ \\
$2.4 \pm 2.4$
\end{tabular}

\begin{tabular}{c}
\hline NASH $(n=7)$ \\
\hline $5.4 \pm 0.5$ \\
$3 / 4$ \\
$34.1 \pm 11.8$ \\
$37.6 \pm 4.1$ \\
$142.4 \pm 30.3$ \\
$87.7 \pm 20.3$ \\
$6.8 \pm 2.8$ \\
$82.0 \pm 36.7$ \\
$43.0 \pm 22.9$ \\
$191.7 \pm 37.3$ \\
$51.2 \pm 32.2$ \\
$5.8 \pm 4.2$ \\
$5.8 \pm 0.8$ \\
$0.8 \pm 0.1$ \\
$2.5 \pm 0.7$ \\
$1.3 \pm 0.2$ \\
$1.0 \pm 0.3$
\end{tabular}

\begin{tabular}{c}
\hline P-Value \\
\hline 0 \\
0.097 \\
0.003 \\
0.001 \\
0.826 \\
0.826 \\
0.558 \\
0.003 \\
0.003 \\
0.826 \\
0.621 \\
0.558 \\
0.003 \\
0.008 \\
0.084 \\
1.000 \\
0.263 \\
\hline
\end{tabular}

Data are presented as mean \pm S.D.

ALT, alanine aminotransferase; AST, aspartate aminotransferase; DBP, diastolic blood pressure; FBG, fasting blood glucose; GGT, gamma glutamyltransferase; HDL, high-density lipoprotein (HDL); LDH, lactate dehydrogenase; LDL, low-density lipoprotein (HDL); NAFL, non-alcoholic simple fatty liver; NAFLD, non-alcoholic fatty liver disease; NAS, NAFLD activity score; NASH, non-alcoholic steatohepatitis; SBP, systolic blood pressure; TC, total cholesterol; TG, triglyceride.

cholesterol, apolipoprotein A and apolipoprotein B in patients were measured in the Department of Laboratory Medicine, Nanjing Drum Tower Hospital, Nanjing University Medical School, Nanjing, China. The liver and cell TG levels were determined using reagent kits (Beijing Puli Lai Company, Beijing, China). Superoxide dismutase, glutathione peroxidase and malondialdehyde were determined by ELISAs (Beyotime).

\section{Immunohistochemistry}

Human liver samples were washed with PBS, fixed in paraformaldehyde for $24 \mathrm{~h}$, embedded in paraffin and then sliced into $4-\mu \mathrm{m}$ sections. The sections were immunostained with the anti-HIF-1 $\alpha$ antibody (diluted $1: 1000 \mathrm{v} / \mathrm{v} ;$ Abcam) and anti-HIF-2 $\alpha$ antibody (diluted $1: 100 \mathrm{v} / \mathrm{v} ;$ Abcam). Brown staining was considered as a positive result. Images were captured using the Panoramic 250 slide scanner (3DHistech Ltd.).

\section{Western blotting and real-time PCR}

Total protein was extracted using RIPA lysis buffer. For insulin-signaling experiments, the primary hepatocytes were pre-treated with $100 \mathrm{nmol} / \mathrm{L}$ insulin for $15 \mathrm{~min}$ (Sigma-Aldrich) before protein extraction. The protein samples were separated by sodium dodecyl sulphatePAGE and transferred onto a polyvinylidene difluoride membrane (Millipore) and incubated with primary antibodies against HIF-1 $\alpha$ (diluted 1:1000 v/v; Abcam), HIF-2 $\alpha$ (diluted 1:100 v/v; Abcam), AKT (diluted 1:1000 v/v; Cell Signaling Technology) and phospho-AKT (Ser473) (pAKT, diluted 1:1000 v/v; Cell Signaling Technology). All samples were evaluated by re-probing with anti-GAPDH (diluted 1:4000 v/v; Cell Signaling Technology) to verify equal protein loading.

Total RNA was isolated using TRIzol reagent (Invitrogen) and reverse-transcribed using the cDNA RT Kit (Takara BIO). Real-time PCR was performed on a Light Cycler 480 (Roche) with the following cycling conditions: $95^{\circ} \mathrm{C}$ for $10 \mathrm{~min}, 45$ cycles of $95^{\circ} \mathrm{C}$ for $15 \mathrm{~s}$ and $60^{\circ} \mathrm{C}$ for $1 \mathrm{~min}$. Relative gene expression was normalised to levels of $\beta$-actin as the housekeeping gene and calculated as $2^{-\Delta \Delta \mathrm{Ct}}$. The primers used are listed in Table 2 .

\section{Inhibition of translation and proteasome inhibition}

To detect protein synthesis levels, primary hepatocytes were respectively plated in two six-well plates and divided into a control group (OT $0 \mathrm{~h}$ ) and an oxygen therapy group (OT $1 \mathrm{~h}$ ), both of which were treated with PA for $24 \mathrm{~h}$, and the OT group was administered oxygen therapy for $1 \mathrm{~h}$. The translation inhibitor cycloheximide (0.1 mg/mL, Sigma) was added to each cell group for 0 , $15,30,45$ or $60 \mathrm{~min}$, respectively, and the proteins were collected for Western blotting. To detect proteasomal https://joe.bioscientifica.com https://doi.org/10.1530/JOE-19-0555
(C) 2020 Society for Endocrinology Published by Bioscientifica Ltd.
Printed in Great Britain 
Table 2 Oligonucleotide sequences of primers used for real-time PCR.

\begin{tabular}{l}
\hline Gene \\
\hline$\beta$-actin (human) \\
HIF-1 $\alpha$ (human) \\
HIF-2 $\alpha$ (human) \\
$\beta$-actin (mouse) \\
Hif-1 $\alpha$ (mouse) \\
Hif-2 $\alpha$ (mouse) \\
Srebp1c (mouse) \\
ChREBP (mouse) \\
Acc (mouse) \\
Fasn (mouse) \\
Scd1 (mouse) \\
Dgat1 (mouse) \\
Dgat2 (mouse) \\
Cidea (mouse) \\
Elovl6 (mouse) \\
Plin2 (mouse)
\end{tabular}

\begin{tabular}{l}
\hline Forward primer \\
\hline CATGTACGTTGCTATCCAGGC \\
GAACGTCGAAAAGAAAAGTCTCG \\
CGGAGGTGTTCTATGAGCTGG \\
CCACAGCTGAGAGGGAAATC \\
TCTCGGCGAAGCAAAGAGTC \\
GAGGAAGGAGAAATCCCGTGA \\
TGACCCGGCTATTCCGTGA \\
AGATGGAGAACCGACGTATCA \\
CTCCCGATTCATAATTGGGTCTG \\
GGAGGTGGTGATAGCCGGTAT \\
TTCTTGCGATACACTCTGGTGC \\
GTGCCATCGTCTGCAAGATTC \\
GCGCTACTTCCGAGACTACTT \\
TGACATTCATGGGATTGCAGAC \\
GAAAAGCAGTTCAACGAGAACG \\
CTTGTGTCCTCCGCTTATGTC
\end{tabular}

\begin{tabular}{l}
\hline Reverse primer \\
\hline CTCCTTAATGTCACGCACGAT \\
CCTTATCAAGATGCGAACTCACA \\
AGCTTGTGTGTTCGCAGGAA \\
AAGGAAGGCTGGAAAAGAGC \\
AGCCATCTAGGGCTTTCAGATAA \\
TATGTGTCCGAAGGAAGCTGA \\
CTGGGCTGAGCAATACAGTTC \\
ACTGAGCGTGCTGACAAGTC \\
TCGACCTTGTTTACTAGGTGC \\
TGGGTAATCCATAGAGCCCAG \\
CGGGATTGAATGTTCTTGTCGT \\
GCATCACCACACACCAATTCAG \\
GGGCCTTATGCCAGGAAACT \\
TGACATTCATGGGATTGCAGAC \\
AGATGCCGACCACCAAAGATA \\
GCAGAGGTCACGGTCTTCAC
\end{tabular}

Acc, acetyl coenzyme A carboxylase; ChREBP, carbohydrate response element binding protein; Cidea, cell death-inducing DFFA-like effector A; Dgat1, diacylglycerol O-acyltransferase 1; Dgat2, diacylglycerol O-acyltransferase 2; Elovl6, ELOVL fatty acid elongase 6; Fasn, fatty acid synthase; HIF-1 $\alpha$, hypoxia-inducible factor-1 $\alpha$; HIF-2 $\alpha$, hypoxia-inducible factor-2 $\alpha$; Plin2, perilipin 2; Scd1, stearoyl-CoA desaturase; Srebp 1c, sterol response element-binding protein 1c.

degradation, primary hepatocytes were divided into the following four groups: CON, OT, MG132 and OT+MG132, which were all treated with PA for $24 \mathrm{~h}$. The OT and OT+MG132 groups were supplied with oxygen for $1 \mathrm{~h}$, and the MG132 and OT +MG132 groups were treated with the proteasomal inhibitor MG132 (0.01 mmol/L, Sigma) for $1 \mathrm{~h}$. The proteins were collected for Western blotting.

\section{Data analysis and statistical calculations}

Statistical analysis was performed with GraphPad (version 7.0, GraphPad, Inc.) and SPSS (version 22.0, SPSS, Inc.). The results are expressed as the mean \pm S.D. and as proportions (qualitative data). The statistical differences were analysed by one-way ANOVA with Tukey's post hoc test for multiple comparisons or chi-square tests for categorical variables. $P<0.05$ was considered statistically significant.

\section{Results}

\section{OT improves hepatic steatosis}

To verify whether OT can improve oxygen flow in the liver, we measured the blood oxygen concentration at the outlet of the inferior vena cava near the liver using an oxygen probe. The results revealed that OT significantly increased the blood oxygen concentration (Fig. 1A) and decreased the body weight and liver/body weight ratios in
HFD-administered mice (Fig. 1B, C, D and E). Hepatic steatosis due to the HFD was improved, as evidenced by decreased hepatic TG levels and reduced sizes and numbers of lipid droplets based on H\&E- and Oil Red O-stained hepatic tissue sections (Fig. 1F and G), which was accompanied by the alleviation of dyslipidaemia (Fig. $1 \mathrm{H}$ ). Furthermore, OT significantly improved hepatocellular steatosis and reduced intracellular TG levels in PA-treated primary hepatocytes $(P<0.05$; Fig. 1 I and J).

We next investigated the effect of OT on glucose metabolism and insulin resistance by performing GTTs and ITTs. The results revealed that OT lowered HFD-induced hyperglycaemia and increased insulin sensitivity (Fig. 2A and B). OT also stimulated the insulin-signaling pathway, as evidenced by the increase in hepatocyte insulin-stimulated Akt phosphorylation levels with PA (Fig. 2H). The levels of oxidative stress indicators, namely malondialdehyde and glutathione peroxidase, in the livers of HFD mice, as well as superoxide dismutase in PA-treated primary hepatocytes, were also decreased by OT (Fig. 2C, D and E). Furthermore, OT increased the proliferation of primary hepatocytes, as determined using CCK8 $(P<0.05$; Fig. $2 \mathrm{~F})$ and reduced the obvious swelling of mitochondria and mitochondrial cleavage in PA-treated primary hepatocytes (Fig. 2G).

\section{HIF- $2 \alpha$ but not HIF-1 $\alpha$ is related to NAFLD progression}

To investigate the association between HIF- $1 \alpha / \mathrm{HIF}-2 \alpha$ and NAFLD, we evaluated the expression of HIF- $1 \alpha$ and HIF- $2 \alpha$ in the liver tissues of non-NAFLD group individuals 
A

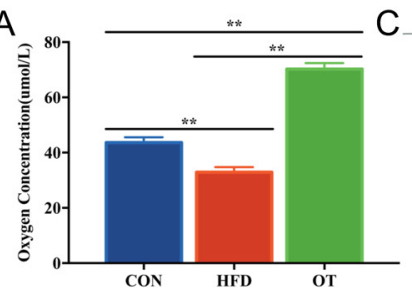

B
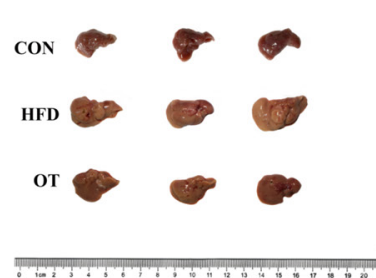

$\mathrm{C}$
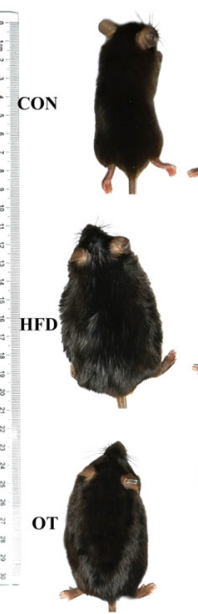

G

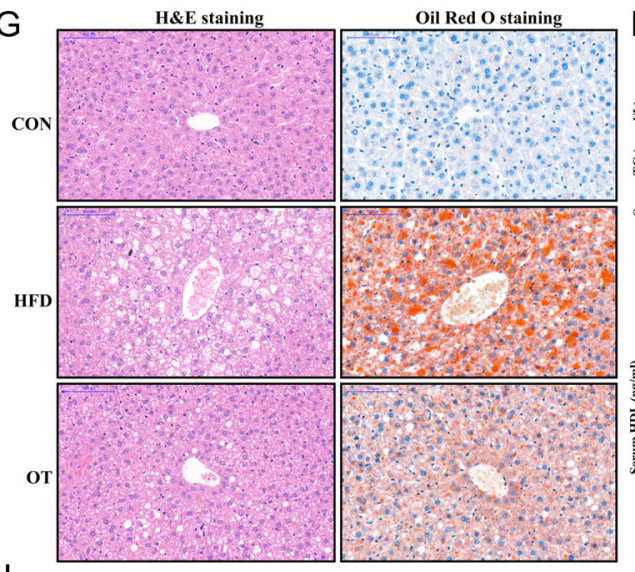

I

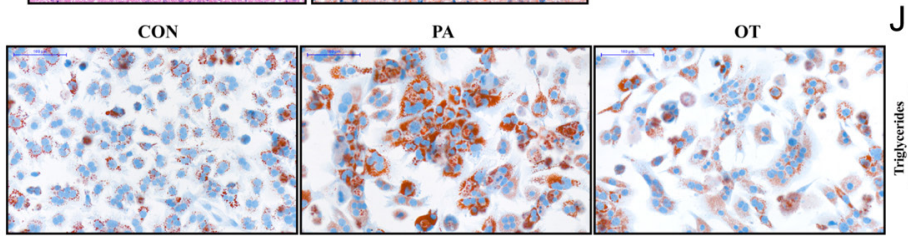

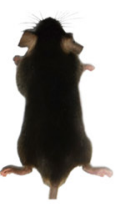
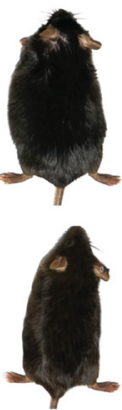

$\mathrm{H}$
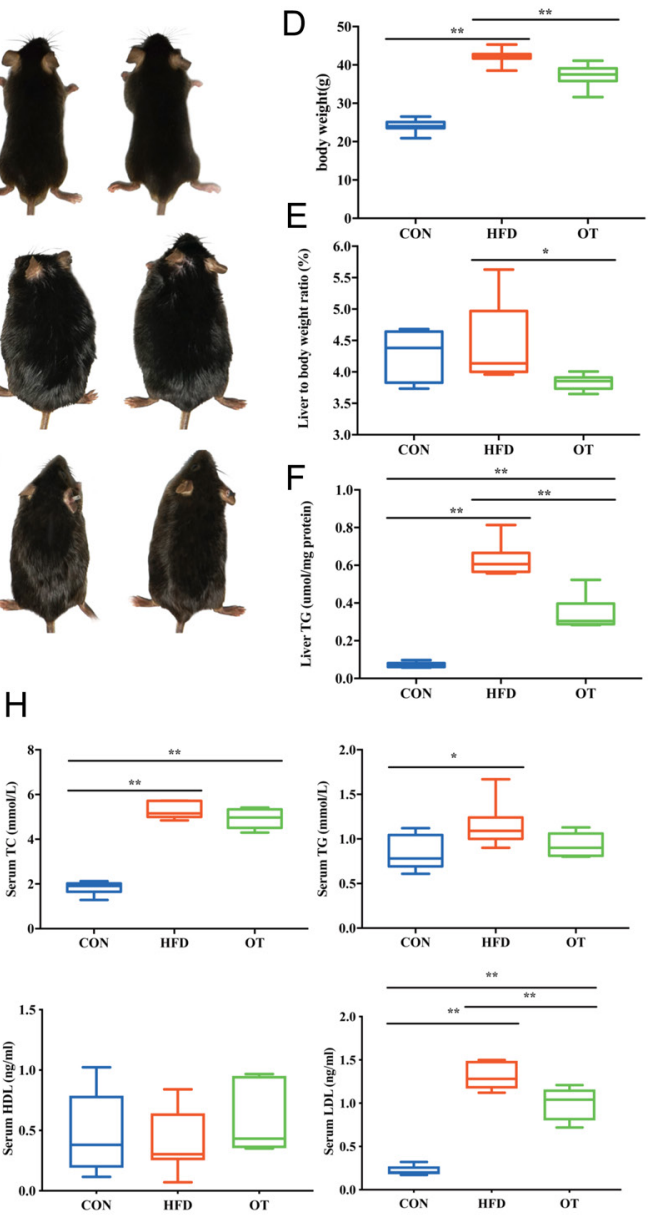

$\mathrm{J}$

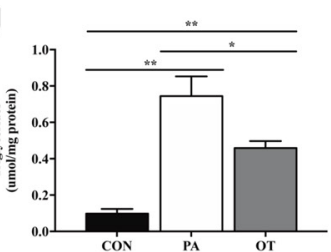

\section{Figure 1}

Oxygen therapy (OT) improves hepatic steatosis in high fat diet (HFD)-fed mice and in palmitic acid (PA)-pre-treated primary mouse hepatocytes. Oxygen content in the inferior vena cava from the CON (control), HFD and OT groups ( $n=3$ subjects/group) (A). Liver morphology (B), gross morphology (C), body weight (D), liver-to-body weight ratio (E) and hepatic triglyceride (TG) levels (F). Representative H\&E- and Oil Red O-stained liver sections (magnification $\times 20)(G)$. Serum total cholesterol (TC), TG, high-density lipoprotein (HDL) and low-density lipoprotein (LDL) levels (H). Oil Red O staining of primary hepatocytes from the CON, PA and OT groups ( $n=3$ subjects/group) (I). Hepatocyte TG levels (J). Values are the mean \pm S.D. ( $n=7$ animals/group). $\star P<0.05, * \star P<0.01$. Data are represented as the mean \pm S.D. A full color version of this figure is available at https://doi.org/10.1530/JOE-19-0555.

(patients with hepatic hemangioma, $n=7$ ) and individuals with NAFLD $(n=14)$ by immunohistochemistry (Fig. 3A and C) and real-time PCR (Fig. 3B). The results suggested that compared to those in patients without NAFLD, patients with NAFLD had significantly higher HIF- $2 \alpha$ levels in the cytoplasm and nuclei of hepatocytes, and the hepatic gene and protein expression of HIF- $2 \alpha$ was associated with the progression of NAFLD. Increased hepatic gene and protein expression of HIF-1 $\alpha$ was confirmed in patients with NAFLD, but it did not correlate with the progression of disease.

\section{OT reduces hepatic lipogenesis and HIF-2 $\alpha$ mediates the alleviation of hepatic steatosis induced by OT}

To further explore how OT might improve hepatic steatosis, we tested lipid synthesis-related genes before and after OT in vivo and in vitro. We found that OT 
A
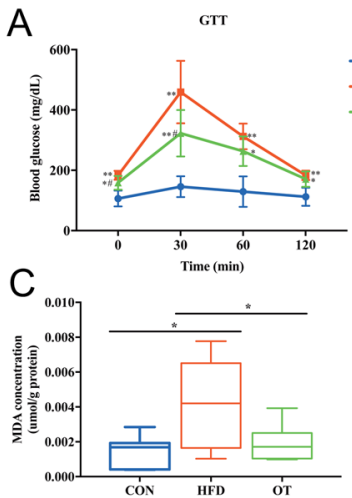

G

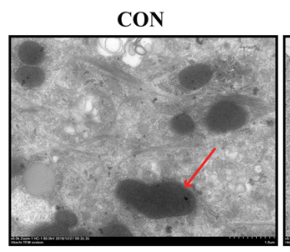

mitochondrial volume density

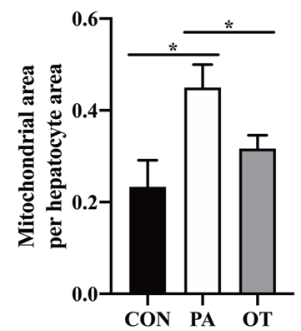

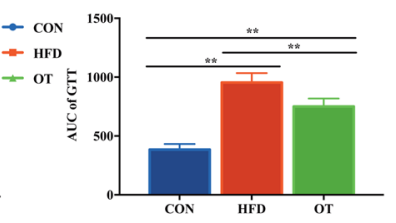

D

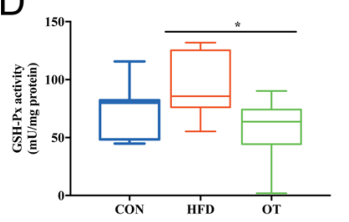

B

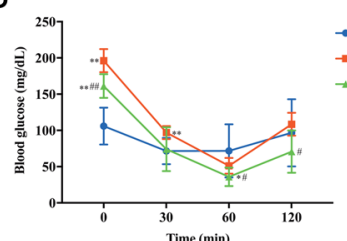

E

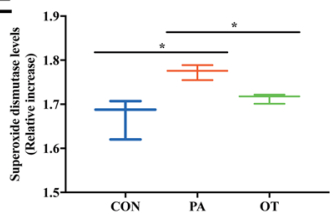

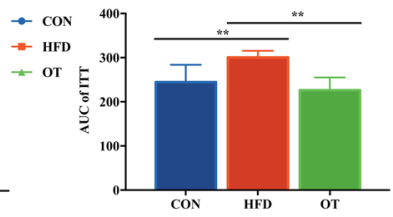

$\mathrm{F}$

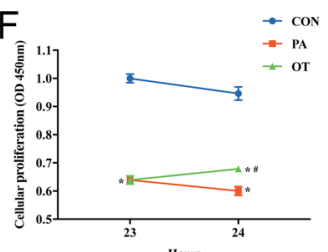

$\mathrm{H}$

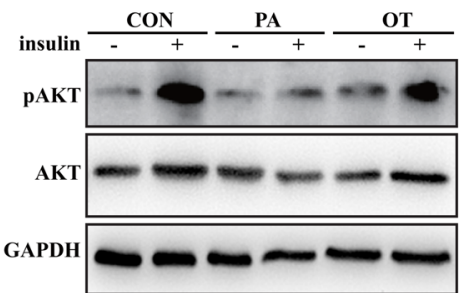

mitochondrial number

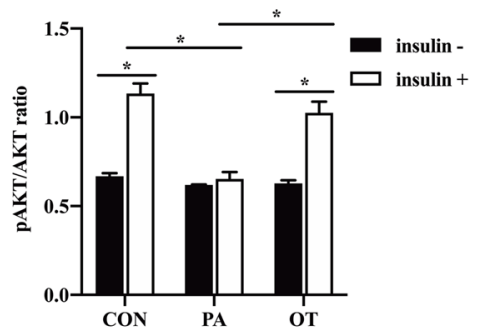

Figure 2

Oxygen therapy (OT) improves glucose metabolism and insulin sensitivity in high fat diet (HFD)-fed mice, alleviates hepatic oxidative stress and improves hepatocellular viability and mitochondrial structure. Glucose tolerance testing (GTT; A) and insulin tolerance testing (ITT; B). *P<0.05, $* * P<0.01$ vs CON (control) group, $\# P<0.05, \# P<0.01$ vs HFD group. Hepatic oxidative stress indicators MDA and GSH-Px in the CON, HFD and OT groups and SOD levels in primary hepatocytes ( $C, D$ and $E)$. $* P<0.05$. Primary hepatocyte proliferation, as measured by CCK8 assays (F). * $P<0.05$, vs CON group, $\# P<0.05$ vs $P A$ (palmitic acid) group. Mitochondrial morphology analysis (arrow) based on transmission electron microscopy images of primary hepatocytes and quantification of mitochondrial volume density and number (G). Scale bar $=5.0 \mu \mathrm{m}$. ${ }^{*} P<0.05$. Western blotting images of AKT and pAKT (Ser473) expression before and after insulin stimulation among CON, PA and OT groups $(\mathrm{H}) .{ }^{*} P<0.05,{ }^{*} P<0.01$. Data are represented as the mean \pm s.D. A full color version of this figure is available at https://doi.org/10.1530/JOE-19-0555.

A

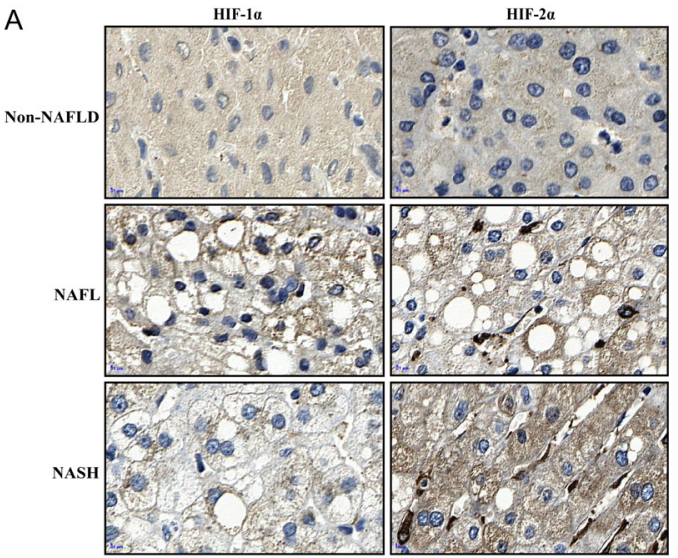

B

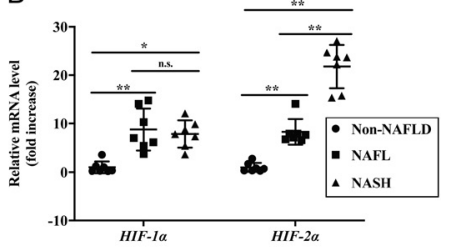

C

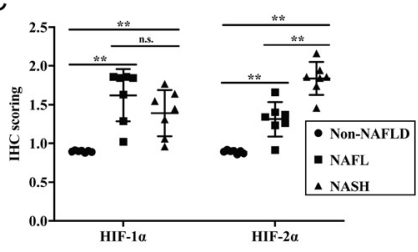

Figure 3

Hepatic HIF-2 $\alpha$ is overexpressed with the development and progression of non-alcoholic fatty liver disease (NAFLD) in humans.

Representative immunohistochemical staining for the expression of HIF-1 $\alpha$ and HIF-2 $\alpha$ in human hepatic tissue from non-NAFLD, NAFL and non-alcoholic steatohepatitis (NASH) patients $(n=7$ subjects/group, magnification $\times 80)(A)$. mRNA expression levels and immunohistochemistry analysis of HIF- $1 \alpha$ and HIF$2 \alpha$ in human hepatic tissue (B and $C$ ). Not significant (n.s.) $P>0.05, * P<0.05, * * P<0.01$. Data are represented as the mean \pm S.D. A full color version of this figure is available at https:// doi.org/10.1530/JOE-19-0555. https://joe.bioscientifica.com https://doi.org/10.1530/JOE-19-0555
(C) 2020 Society for Endocrinology Published by Bioscientifica Ltd.
Printed in Great Britain 
down-regulated genes that are associated with hepatic de novo lipogenesis in HFD mice, such as sterol response element-binding protein 1c (Srebp1c), carbohydrate response element binding protein (ChREBP), acetyl coenzyme A carboxylase $(A c c)$, fatty acid synthase (Fasn), stearoyl-CoA desaturase (Scd1), diacylglycerol O-acyltransferase (Dgat) 1, Dgat2, cell death-inducing DFFAlike effector A (Cidea), ELOVL fatty acid elongase 6 (Elovl6) and perilipin 2 (Plin2), and similar results were obtained in vitro (Fig. 4A). We also found that OT decreased the hepatic gene and protein expression of HIF-2 $\alpha$ due to HFD and PA in vivo and in vitro (Fig. $4 \mathrm{~B}$ and $\mathrm{C}$ ). To verify whether the reduction in HIF- $2 \alpha$ is a key molecular mechanism through which OT alleviates hepatic steatosis, HIF-2 $\alpha$ overexpression in primary hepatocytes was achieved through lentivirus transduction. The results showed that the improvement in hepatic steatosis mediated by
OT was diminished by the overexpression of HIF-2 $\alpha$, as shown by Oil Red O staining (Fig. 4D) and TG levels (Fig. $4 \mathrm{E})$. Furthermore, the overexpression of HIF-2 $\alpha$ inhibited the suppressive effect of OT on genes associated with hepatic de novo lipogenesis in primary hepatocytes treated with PA (Fig. 4F). Finally, OT was confirmed to decrease HIF- $2 \alpha$ levels by reducing the synthesis of HIF- $2 \alpha$ and promoting the ubiquitination/degradation of HIF- $2 \alpha$ in primary hepatocytes (Fig. 4G and H) and thus inhibited hepatic lipogenesis.

\section{Discussion}

HIF- $2 \alpha$ activation, promoted by hepatic hypoxia, plays a pivotal role in hepatic steatosis. In the present study, we verified that OT effectively and safely improves
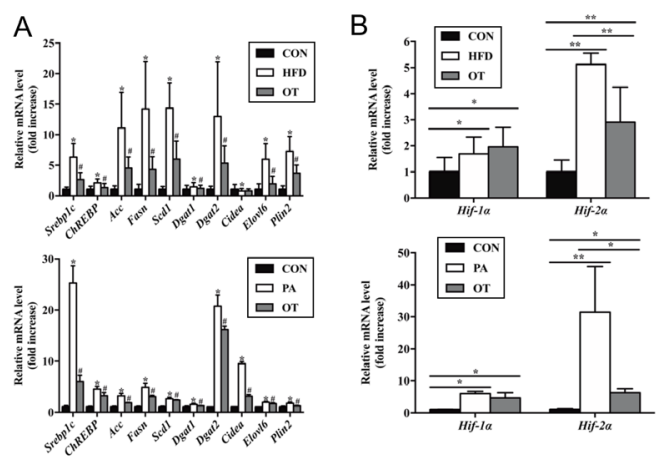

D

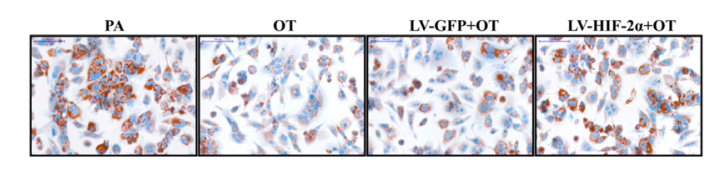

$\mathrm{F}$

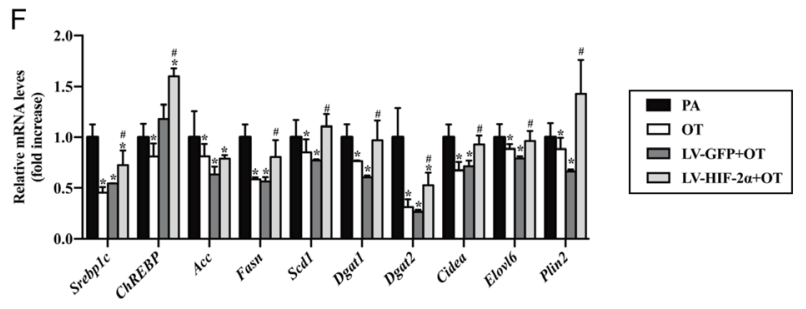

G

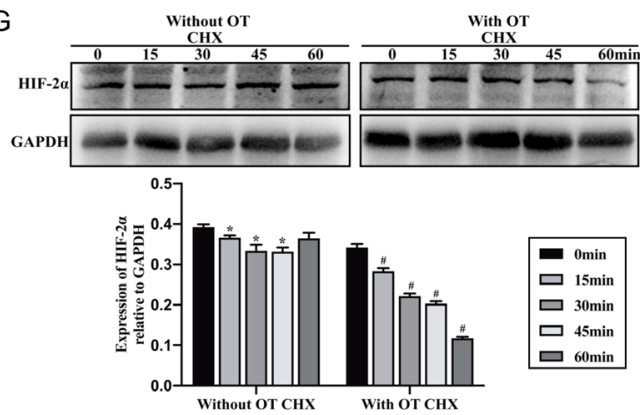

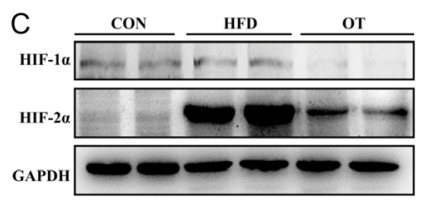
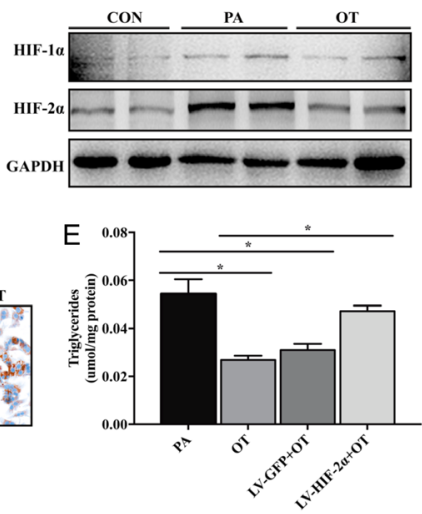

$\mathrm{H}$

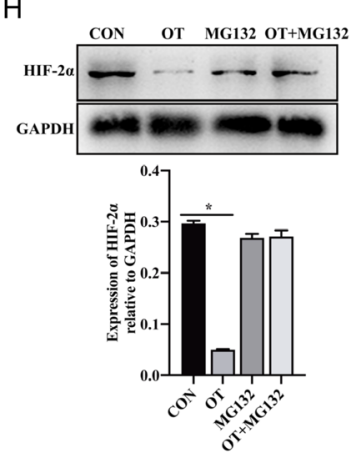

Figure 4

Oxygen therapy (OT) down-regulates gene and protein levels related to hepatic lipogenesis and $\mathrm{HIF}-2 \alpha$ in vivo and in vitro, and HIF- $2 \alpha$ overexpression inhibits the effect of OT on hepatic steatosis and lipogenesis. Expression of hepatic lipogenesis genes in mice and primary hepatocytes (A). ${ }^{\star} P<0.05$ vs CON (control) group, $\# P<0.05$ vs high-fat diet (HFD) or palmitic acid (PA) group. Hepatic gene (B) and protein (C) expression levels of HIF- $1 \alpha$ and HIF- $2 \alpha$ in mice and primary hepatocytes. ${ }^{*} P<0.05, * \star P<0.01$. Oil Red $\mathrm{O}$ staining (D) and hepatocyte triglyceride levels (E) in the PA, OT, LV-GFP + OT and LV-HIF- $2 \alpha+$ OT groups of primary hepatocytes ( $n=3$ subjects/ group). ${ }^{*} p<0.05, * * p<0.01$. Hepatic expression levels of hepatic lipogenesis genes among PA, OT, LV-GFP + OT or LV-HIF- $2 \alpha+$ OT groups $(n=4$ subjects/group) (F). * $P<0.05$ vs $P A$ group, $\# P<0.05$ vs OT group. The degradation rate constants of HIF- $2 \alpha$ proteins at $0,15,30,45$ and 60 min after cycloheximide ( $\mathrm{CHX})$ treatment with or without OT in primary hepatocytes $(G) * P<0.05$ vs 0 min without OT, $\# P<0.05$ vs 0 min with OT. The protein levels of HIF-2 $\alpha$ with MG132, OT or MG132 and OT in primary hepatocytes $(H)$. $\star P<0.05$. Data are represented as the mean \pm S.D. A full color version of this figure is available at https://doi.org/10.1530/JOE-19-0555. 
HFD-induced NAFLD and glucolipid metabolism disorders in a mouse model, as well as hepatic steatosis induced by PA. Decreased hepatic HIF- $2 \alpha$ expression induced by OT might thus be beneficial.

Hepatic hypoxia facilitates and aggravates NAFLD. In a previous study, $o b / o b$ mice developed NAFLD at an earlier age when raised in an environment of chronic intermittent hypoxic environment (Li et al. 2005). Furthermore, exposure to a hypoxic atmosphere was found to accelerate the progression of NASH in hepatocellular Pten knockout mice and HFD-fed C57BL/6 mice (Savransky et al. 2007, Piguet et al. 2009). Hepatic cells cultured under a hypoxic environment also exhibit steatosis (Savransky et al. 2007, Piguet et al. 2009). We found that hepatic hypoxia and hepatic steatosis in NAFLD mice caused by HFD were improved by OT, and accordingly, improvements in the hepatocyte insulin-signaling pathway induced by OT play a role in hepatic steatosis.

Hypoxia up-regulates the hepatic lipogenesis-related genes Srebp1c and Acc in obese mice with hepatic steatosis and activates pro-inflammatory cytokines interleukin (IL) 1 and IL6 in NASH mice (Li et al. 2005, Piguet et al. 2009). We found that OT decreased the expression of hepatic de novo lipogenesis genes in NAFLD mice and PA-pre-treated primary hepatocytes, indicating that it can suppress hepatic steatosis by reducing hepatic lipid synthesis, thereby inhibiting the progression of NAFLD.

HIF- $1 \alpha$ and HIF- $2 \alpha$ are both key messenger proteins that mediate hepatocyte metabolism upon hypoxia exposure. HIF-1 $\alpha$ is mainly involved in the short-term severe hypoxia response and HIF- $2 \alpha$ is a major anoxic response factor involved in long-term mild hypoxia,

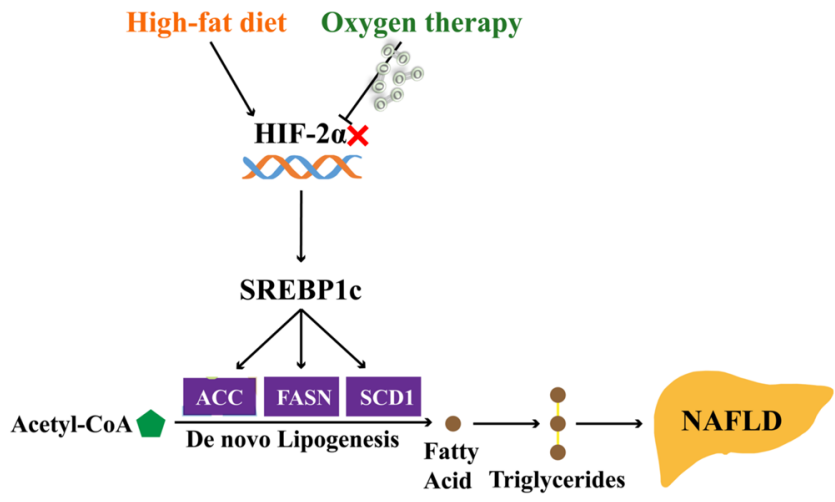

\section{Figure 5}

Schematic representation of the mechanisms underlying the effects of oxygen therapy on non-alcoholic fatty liver disease (NAFLD). Oxygen therapy alleviates hepatic lipogenesis and steatosis by inhibiting HIF-2 $\alpha$. A full color version of this figure is available at https://doi.org/10.1530/ JOE-19-0555. such as NAFLD (Koh \& Powis 2012). Our findings are similar to those of other studies, that is, hepatic HIF- $2 \alpha$ and not HIF- $1 \alpha$ is specifically increased and overexpressed during the development and progression of NAFLD in humans and mice and that HIF- $2 \alpha$ expression is increased in lipid droplet-containing hepatocytes induced by PA (Xie et al. 2017, Morello et al. 2018). We also found that OT suppressed hepatic HIF-2 $\alpha$ expression and improved hepatic steatosis in vivo and in vitro. Furthermore, we confirmed that OT decreases the synthesis of HIF- $2 \alpha$ and promotes the ubiquitination/degradation of HIF- $2 \alpha$ in vitro. Conversely, up-regulating HIF- $2 \alpha$ attenuated the OT-mediated effects of alleviating lipid deposition and suppressing lipogenesis in vitro. Thus, hypoxia following increased lipid droplet accumulation in hepatocytes might induce the overexpression of HIF- $2 \alpha$, further promoting lipid deposition and aggravating hepatic hypoxia. This cycle could perpetuate the development and progression of NAFLD and can be disrupted by OT.

Hyperbaric (2.5 atm) OT slightly improves hepatic steatosis in NASH mice but aggravates inflammation and promotes aberrant TNF $\alpha$ expression. However, liver oxidative stress was found to be relieved by this treatment in ApoE-knockout mice in other studies (Kudchodkar et al. 2007, Tsuneyama et al. 2011). In the present study, we confirmed that $1 \mathrm{~atm}$ of OT alleviates hepatic oxidative stress and improves hepatocellular viability and mitochondrial structure. This indicated that although OT relieves hepatic steatosis, a suitable concentration and pressure of OT should be determined to avoid aggravating hepatic inflammation. In addition, although we found that OT inhibited hepatic de novo lipogenesis by reducing HIF- $2 \alpha$ expression and improved glycometabolism and the hepatic insulin signaling pathway, the detailed mechanism underlying this effect requires further analysis. Another limitation of the present study was the lack of a local liver-targeting OT experiment; thus, we could not exclude the fact that general OT might also affect NAFLD by improving hypoxia in other organs. Finally, we did not detect mitochondrial respiratory rates in the present study; however, in other studies, up-regulated hepatic mitochondrial respiration was confirmed in patients with NAFLD and in HFD-diet-induced NAFLD mice, and the development of mitochondrial dysfunction with the progression of NAFLD was clarified in these studies. The results indicated higher hepatocyte oxygen consumption in the early phase of NAFLD and a loss of hepatic mitochondrial functional adaptation with the progression of NAFLD (Anavi et al. 2017). The obvious swelling of the mitochondria and mitochondrial cleavage 
in PA-treated primary hepatocytes in the present study support the previous results.

In conclusion, we found that OT safely and effectively improves hepatic hypoxia and steatosis, improves glucolipid disorders related to NAFLD in HFD mice, and decreases lipid droplets in hepatocytes pre-treated with PA. The inhibition of hepatic HIF- $2 \alpha$ expression mediated by OT might thus play a vital role in the treatment of NAFLD (Fig. 5). We determined the effect of OT on NAFLD, indicating that OT can be used as a new and effective therapy for NAFLD.

\section{Declaration of interest}

The authors declare that there is no conflict of interest that could be perceived as prejudicing the impartiality of the research reported.

\section{Funding}

This work was supported by the National Key Research and Development Program of China (grant numbers 2016YFC1304804 and 2017YFC1309605), the National Natural Science Foundation of China Grant Awards (grant numbers 81570736, 81570737, 81600632, 81600637, 81703294, 81770819, $81800719,81800752,81970689,81970704$ and 81900787), the Key Research and Development Program of Jiangsu Province of China (grant numbers BE2015604 and BE2016606), the Jiangsu Provincial Key Medical Discipline (grant numbers ZDXKB2016012), the Six Talent Peaks Project of Jiangsu Province of China (grant numbers YY-086), the Jiangsu Provincial Medical Talent (grant numbers ZDRCA2016062), the Fundamental Research Funds for the Central Universities (grant numbers 021414380444), the Scientific Research Project of the Fifth Phase of '333 Project' of Jiangsu Province of China, the Health technology development fund project of Nanjing, China (grant numbers YKK18067) and the Key Project of Nanjing Clinical Medical Science.

\section{Author contribution statement}

$\mathrm{L} Y$ contributed to the study design, data collection and analysis and drafting of the manuscript. H R W contributed to the technical support of oxygen therapy. $\mathrm{XX} \mathrm{H}$ and $\mathrm{H} \mathrm{H} \mathrm{L} \mathrm{contributed} \mathrm{to} \mathrm{the} \mathrm{technical} \mathrm{support}$ of animal experiments. $\mathrm{D} \mathrm{L} \mathrm{Z} \mathrm{contributed} \mathrm{to} \mathrm{the} \mathrm{data} \mathrm{interpretation.}$ J H W contributed to the study design and the technical support of oxygen therapy. Y B and W H F contributed to the study design and manuscript revision. The final version of the manuscript was read and approved by all authors.

\section{Acknowledgements}

The authors thank Editage, a brand of Cactus Communications, who offered professional English language editing.

\section{REFERENCES}

Anavi S, Madar Z \& Tirosh O 2017 Non-alcoholic fatty liver disease, to struggle with the strangle: oxygen availability in fatty livers. Redox Biology 13 386-392. (https://doi.org/10.1016/j.redox.2017.06.008) Birchmeier W 2016 Orchestrating Wnt signalling for metabolic liver zonation. Nature Cell Biology 18 463-465. (https://doi.org/10.1038/ ncb3349)

(c) 2020 Society for Endocrinology Published by Bioscientifica Ltd. Printed in Great Britain
Byrne CD \& Targher G 2015 NAFLD: a multisystem disease. Journal of Hepatology 62 (Supplement) S47-S64. (https://doi.org/10.1016/j. jhep.2014.12.012)

Cohen JC, Horton JD \& Hobbs HH 2011 Human fatty liver disease: old questions and new insights. Science 332 1519-1523. (https://doi. org/10.1126/science.1204265)

Febbraio MA, Reibe S, Shalapour S, Ooi GJ, Watt MJ \& Karin M 2019 Preclinical models for studying NASH-driven HCC: how useful are they? Cell Metabolism 29 18-26. (https://doi.org/10.1016/j. cmet.2018.10.012)

Hannah Jr WN \& Harrison SA 2016 Lifestyle and dietary interventions in the management of nonalcoholic fatty liver disease. Digestive Diseases and Sciences 61 1365-1374. (https://doi.org/10.1007/s10620-0164153-y)

Kietzmann T 2017 Metabolic zonation of the liver: the oxygen gradient revisited. Redox Biology 11 622-630. (https://doi.org/10.1016/j. redox.2017.01.012)

Kietzmann T 2019 Liver zonation in health and disease: hypoxia and hypoxia-inducible transcription factors as concert masters. International Journal of Molecular Sciences 20 2347. (https://doi. org/10.3390/ijms20092347)

Koh MY \& Powis G 2012 Passing the baton: the HIF switch. Trends in Biochemical Sciences 37 364-372. (https://doi.org/10.1016/j. tibs.2012.06.004)

Koliaki C, Szendroedi J, Kaul K, Jelenik T, Nowotny P, Jankowiak F, Herder C, Carstensen M, Krausch M, Knoefel WT, et al. 2015 Adaptation of hepatic mitochondrial function in humans with non-alcoholic fatty liver is lost in steatohepatitis. Cell Metabolism 21 739-746. (https://doi.org/10.1016/j.cmet.2015.04.004)

Kudchodkar BJ, Pierce A \& Dory L 2007 Chronic hyperbaric oxygen treatment elicits an anti-oxidant response and attenuates atherosclerosis in apoE knockout mice. Atherosclerosis 193 28-35. (https://doi.org/10.1016/j.atherosclerosis.2006.08.018)

Li J, Grigoryev DN, Ye SQ, Thorne L, Schwartz AR, Smith PL, O'Donnell CP \& Polotsky VY 2005 Chronic intermittent hypoxia upregulates genes of lipid biosynthesis in obese mice. Journal of Applied Physiology 99 1643-1648. (https://doi.org/10.1152/ japplphysiol.00522.2005)

Mantena SK, Vaughn DP, Andringa KK, Eccleston HB, King AL, Abrams GA, Doeller JE, Kraus DW, Darley-Usmar VM \& Bailey SM 2009 High fat diet induces dysregulation of hepatic oxygen gradients and mitochondrial function in vivo. Biochemical Journal 417 183-193. (https://doi.org/10.1042/BJ20080868)

Morello E, Sutti S, Foglia B, Novo E, Cannito S, Bocca C, Rajsky M, Bruzzi S, Abate ML, Rosso C, et al. 2018 Hypoxia-inducible factor 2alpha drives nonalcoholic fatty liver progression by triggering hepatocyte release of histidine-rich glycoprotein. Hepatology 67 2196-2214. (https://doi.org/10.1002/hep.29754)

Pais R, Barritt AST, Calmus Y, Scatton O, Runge T, Lebray P, Poynard T, Ratziu V \& Conti F 2016 NAFLD and liver transplantation: current burden and expected challenges. Journal of Hepatology 65 1245-1257. (https://doi.org/10.1016/j.jhep.2016.07.033)

Piguet AC, Stroka D, Zimmermann A \& Dufour JF 2009 Hypoxia aggravates non-alcoholic steatohepatitis in mice lacking hepatocellular PTEN. Clinical Science 118 401-410. (https://doi org/10.1042/CS20090313)

Qu A, Taylor M, Xue X, Matsubara T, Metzger D, Chambon P, Gonzalez FJ \& Shah YM 2011 Hypoxia-inducible transcription factor 2alpha promotes steatohepatitis through augmenting lipid accumulation, inflammation, and fibrosis. Hepatology 54 472-483. (https://doi. org/10.1002/hep.24400)

Ramakrishnan SK \& Shah YM 2017 A central role for hypoxia-inducible factor (HIF)-2alpha in hepatic glucose homeostasis. Nutrition and Healthy Aging 4 207-216. (https://doi.org/10.3233/NHA-170022)

Ramakrishnan SK, Zhang H, Takahashi S, Centofanti B, Periyasamy S, Weisz K, Chen Z, Uhler MD, Rui L, Gonzalez FJ, et al. 2016 HIF2alpha is an essential molecular brake for postprandial 
hepatic glucagon response independent of insulin signaling. Cell Metabolism 23 505-516. (https://doi.org/10.1016/j. cmet.2016.01.004)

Romero-Gomez M, Zelber-Sagi S \& Trenell M 2017 Treatment of NAFLD with diet, physical activity and exercise. Journal of Hepatology 67 829-846. (https://doi.org/10.1016/j.jhep.2017.05.016)

Rotman Y \& Sanyal AJ 2017 Current and upcoming pharmacotherapy for non-alcoholic fatty liver disease. Gut 66 180-190. (https://doi. org/10.1136/gutjnl-2016-312431)

Savransky V, Bevans S, Nanayakkara A, Li J, Smith PL, Torbenson MS \& Polotsky VY 2007 Chronic intermittent hypoxia causes hepatitis in a mouse model of diet-induced fatty liver. American Journal of Physiology: Gastrointestinal and Liver Physiology 293 G871-G877. (https://doi.org/10.1152/ajpgi.00145.2007)
Tsuneyama K, Chen YC, Fujimoto M, Sasaki Y, Suzuki W, Shimada T, Iizuka S, Nagata M, Aburada M \& Chen SY 2011 Advantages and disadvantages of hyperbaric oxygen treatment in mice with obesity hyperlipidemia and steatohepatitis. ScientificWorldJournal 11 2124-2135. (https://doi.org/10.1100/2011/380236)

Wei K, Piecewicz SM, Mcginnis LM, Taniguchi CM, Wiegand SJ, Anderson K, Chan CW, Mulligan KX, Kuo D, Yuan J, et al. 2013 A liver Hif-2alpha-Irs2 pathway sensitizes hepatic insulin signaling and is modulated by vegf inhibition. Nature Medicine 19 1331-1337. (https://doi.org/10.1038/nm.3295)

Xie C, Yagai T, Luo Y, Liang X, Chen T, Wang Q, Sun D, Zhao J, Ramakrishnan SK, Sun L, et al. 2017 Activation of intestinal hypoxiainducible factor 2alpha during obesity contributes to hepatic steatosis. Nature Medicine 23 1298-1308. (https://doi.org/10.1038/nm.4412)

Received in final form 22 April 2020

Accepted 29 April 2020

Accepted Manuscript published online 5 May 2020
(C) 2020 Society for Endocrinology Published by Bioscientifica Ltd.
Printed in Great Britain 